\title{
Implantable access system for prolonged intrapleural immunotherapy
}

\author{
P. Driesen*, C. Boutin*, J.R. Viallat**, P.H. Astoul*, J.P. Vialette*, J. Pasquier**
}

Implantable access system for prolonged intrapleural immunotherapy. P. Driesen, $C$. Boutin, J.R. Viallat, P.H. Astoul, J.P. Vialette, J. Pasquier. CERS Journals Ltd 1994.

ABSTRACT: We describe our experience using an implantable Port-A-Cath access system for intrapleural administration of $\gamma$-interferon $(\gamma$-IFN) in malignant mesothelioma patients.

Twenty nine patients, with histologically proven malignant mesotheliomas were included in this study. To avoid complications the device was implanted in a subcutaneous pocket, and the catheter was connected via a tunnel. Also, a suction drain was installed in the pocket after placement.

This procedure greatly reduced the high infection rate $(64 \%)$ encountered with conventional open chest tubes. Patients' tolerance was excellent and maintenance minimal.

In our opinion, the Port-A-Cath system is the most suitable device for intracavitary long-term therapy of malignant pleural effusions. Eur Respir J., 1994, 7, 1889-1892.

\author{
*Dept of Pulmonology, Hôpital de la \\ Conception, Marseille, France. **Institut \\ Paoli-Calmette, Marseille, France.
}

Correspondence: C. Boutin, Dept of Pulmonology, Hôpital de la Conception 13385 Marseille Cedex 5, France.

Keywords: Cytokines

intrapleural immunotherapy

mesothelioma

Port-A-Cath

thoracoscopy

Received: May 71993

Accepted for publication January 261994
Since 1989, we have been participating in a multicentre study to determine the effectiveness of immunotherapy with recombinant $\gamma$-interferon $(\gamma$-IFN) in the treatment of patients with early-stage malignant mesothelioma [1]. The study protocol required intrapleural administration of 40 million international units of $\gamma$-IFN twice a week for eight weeks. As a consequence of prolonged open chest tube drainage, local infection was frequent in the early stages of study at all participating centres. This complication occurred in $64 \%$ of our initial 18 patients. To avoid this problem, we decided to use an implantable access system. The purpose of this study was to assess the efficacy and tolerance of the Port-A-Cath ${ }^{\circledR}$ (PAC) (Pharmacia Deltec Inc., USA) for repeat intrapleural instillation of cytokines.

\section{Patients}

The study included 29 patients with histologically proven malignant mesothelioma. The mean age of patients was 60 yrs (range 47-69 yrs). There were 26 males (mean age 53 yrs) and 3 females (mean age 62 yrs). All patients had a Karnofsky performance status of $60-100 \%$, had received no previous treatment, and had an expected survival of more then 3 months. Patients with fever, brain metastases, leucocyte count lower than 3,000 cells $\cdot \mu^{-1}$, serum creatinine level greater than $1.5 \mathrm{ng} \cdot \mathrm{dl}^{-1}$, or serum bilirubin level greater than $1.5 \mathrm{ng} \cdot \mathrm{dl}^{-1}$ were ineligible. Each patient gave written informed consent.

All patients included in this study underwent thoracoscopy with multiple biopsies (more than 20). If gross examination suggested malignant mesothelioma, the PAC was implanted at the end of the procedure. If not, the device was implanted after diagnosis was confirmed by histology.

\section{Device}

The PAC was designed for repeat access to the vascular system. It consists of a stainless steel portal chamber, a silicone catheter and a cath-shield connector to firmly connect the catheter to the chamber.

\section{Method}

After local anaesthesia of the placement site with $10 \mathrm{ml}$ of $2 \%$ lidocaine, an incision approximately $2 \mathrm{~cm}$ long was made and a pocket was created $1 \mathrm{~cm}$ below the skin surface by blunt dissection. The PAC was placed over a rib to provide support during injection. In most cases, the most suitable location was the third or fourth rib on the midclavicular line (fig. 1).

The next steps were guide wire insertion and pleural catheter placement. A trocar was used to make a tunnel, approximately $5 \mathrm{~cm}$ long, from the pocket to the site of entry and to puncture the parietal pleura. When the PAC was placed during thoracoscopy, pleural puncture and guidewire insertion could be performed under visual control. Direct insertion of the guidewire into the pleura was also possible without thoracoscopy if pleural fluid was present. However, if thoracoscopy was not used and there was no pleural fluid, the pleural space had first 


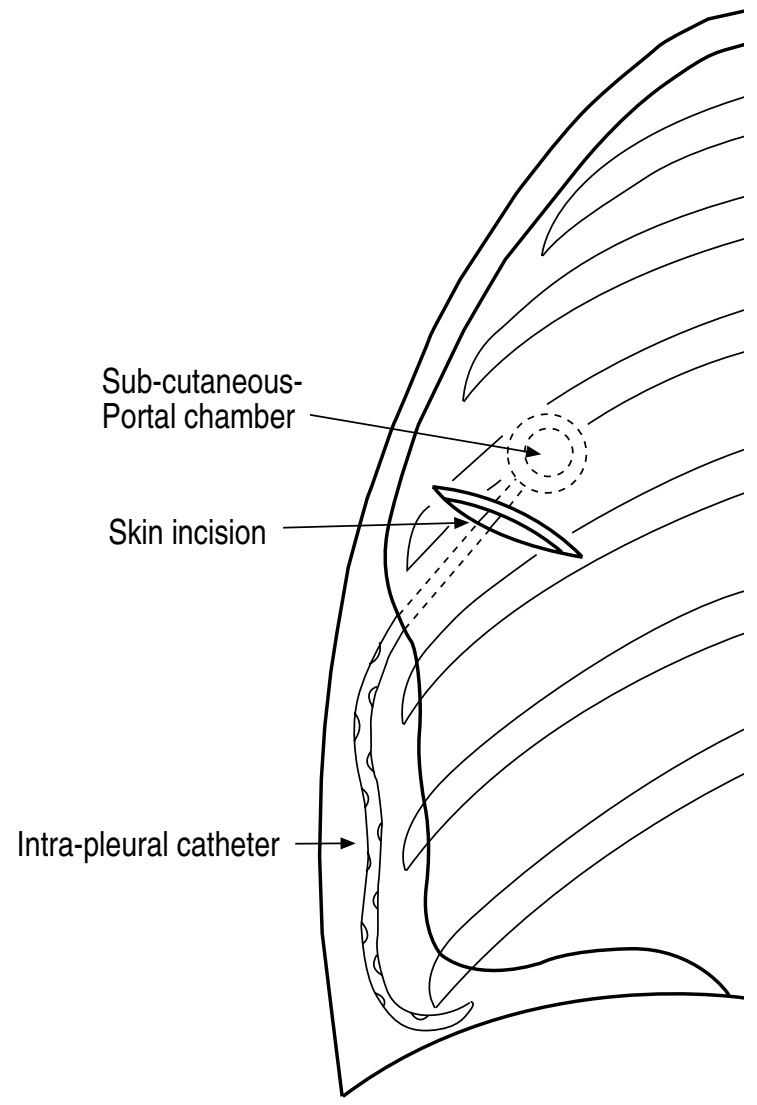

Fig. 1. - Schematic placement of the Port-A-Cath (PAC) system and of the pleural catheter.

to be punctured with a $2 \mathrm{~mm}$ diameter trocar. In this case, penetration of the trocar into the pleural space was detected by negative pressure readings on a manometer during breathing. The guidewire was introduced into the pleural space through the trocar, which was then removed. After insertion of the guide wire, the pleural catheter was threaded under the skin into the pleural cavity.
Finally the PAC was placed in the subcutaneous pocket and its corners were sutured to the underlying muscle fascia. The catheter was attached by the cath-shield connector. A small suction drain with several openings was placed in the pocket to prevent any fluid collection. The pocket was then closed. The PAC should not be under the incision (figs. 1 and 2).

\section{Injection and maintenance}

The treatment could be started as soon as the incision had healed. After cleansing the skin with an antiseptic, injection was performed with a Huber needle. To avoid injection of the subcutaneous tissue, the needle should be inserted through the top of the dome to the bottom of the chamber. To maintain the patency of the PAC, the PAC and catheter should be flushed with heparinized saline once every month.

\section{Results}

In 18 of the 29 patients $(62 \%)$ in this study, no complications occurred. In four patients (14\%), placement of the PAC was followed by transient local oedema, but this did not interfere with treatment. In seven patients (24\%), placement of the PAC was complicated by local supuration (table 1). One of these patients developed an empyema. The offending organism was Staphylococcus aureus in three cases, Staphylococcus epidermidis in two, Pseudomonas aeruginosa in one, and Bacillus sp. in one. In one patient, infection responded to antibiotics and $\gamma$-IFN treatment was completed. In the remaining six patients, removal of the device was necessary. The interval between placement of the device and diagnosis of local infection was variable. All but one infected patient first developed local haematoma immediately after the placement of the PAC.

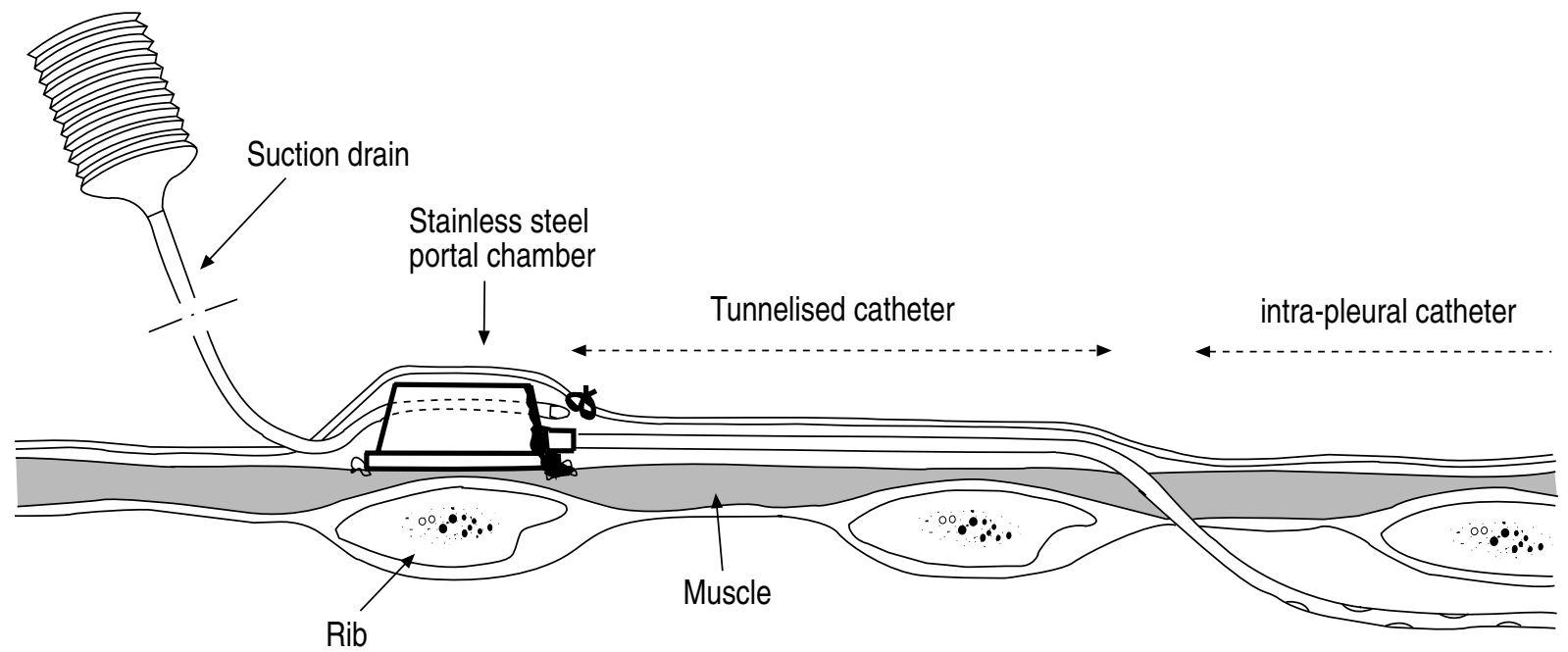

Pleural cavity

Fig. 2. - Port-A-Cath (PAC) placement. The portal chamber was placed under the skin after a $2 \mathrm{~cm}$ incision had been made. The external part of the pleural catheter was tunnelled over $4-5 \mathrm{~cm}$ and connected to the cath-shield connector. 
Table 1. - Local supuration induced by Port-A-Cath system

\begin{tabular}{lcccccc}
\hline Patient & $\begin{array}{c}\text { Date of } \\
\text { placement }\end{array}$ & $\begin{array}{c}\text { Interval* } \\
\text { weeks }\end{array}$ & $\begin{array}{c}\text { Local } \\
\text { haematoma }\end{array}$ & $\begin{array}{c}\text { Cultured } \\
\text { pathogen }\end{array}$ & $\begin{array}{c}\text { Empyema } \\
\text { Removal of } \\
\text { device }\end{array}$ \\
\hline 1. BR & $11 / 89$ & 1 & No & $\begin{array}{c}\text { Staphylococcus aureus } \\
\text { Pseudomonas aeruginosa }\end{array}$ & $\begin{array}{c}\text { No } \\
\text { No }\end{array}$ & Yes \\
2. DS & $11 / 89$ & 2 & Yes & Staphylococcus aureus & Yo & Yes \\
3. BV & $1 / 90$ & 7 & Yes & Staphylococcus aureus & No & Yes \\
4. MP & $2 / 90$ & 7 & Yes & Yes & Bacillus spp & Yes \\
5. SJ & $3 / 90$ & 4 & Yes & Staphylococcus epidermidis & No & Yes \\
6. VF & $3 / 90$ & 6 & Yes & Staphylococcus epidermidis & No \\
7. VL & $3 / 90$ & 5 & & & &
\end{tabular}

*: interval between placement and infection.

a)

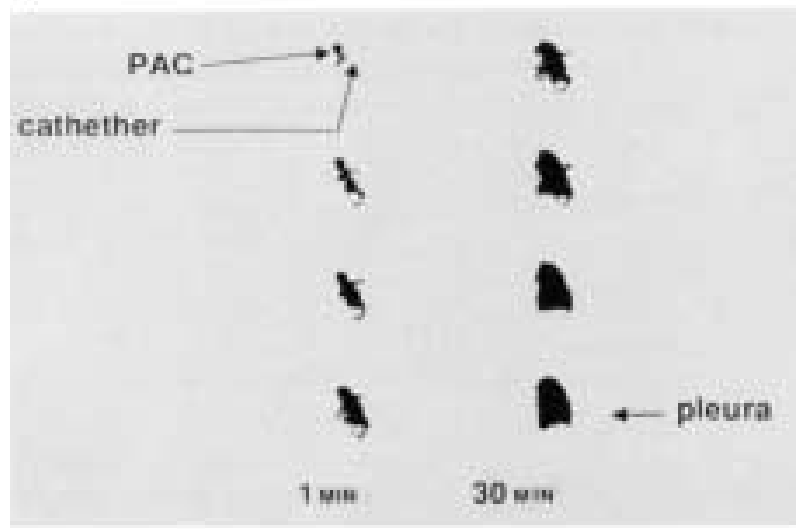

b)

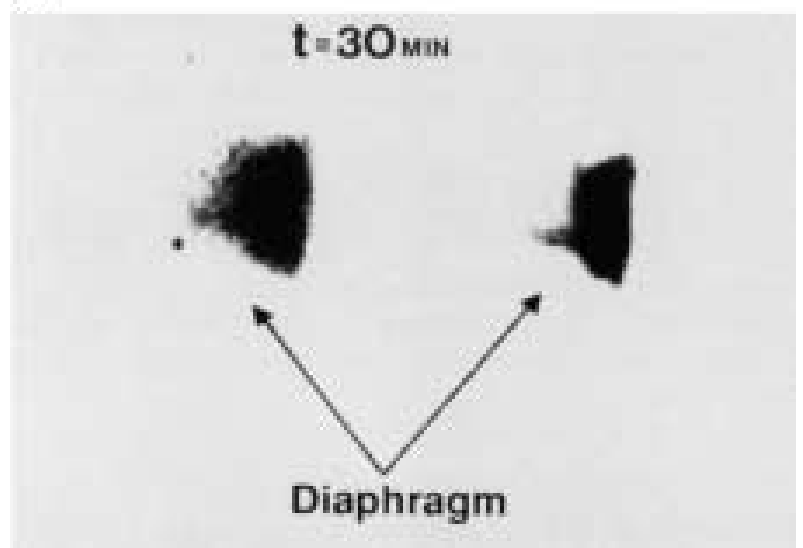

Fig. 3. - Anterior scintigrams of the left pleura. a) Intracavitary injection of $99 \mathrm{~m}$-technetium-labelled macroaggregates through the PortA-Cath (PAC) shows a complete and instant diffusion before $\gamma$-interferon $(\gamma$-IFN) treatment. b) After the end of treatment, the pleural cavity is still adhesion-free.

All seven infectious complications occurred prior to March 1990. Since then, we have routinely placed a drain in the subcutaneous pocket after PAC placement to prevent fluid accumulation. Neither local haematoma nor infection was observed in any of the remaining 14 patients. After treatment, the PAC is easily removed.

\section{Discussion}

A variety of methods have been proposed for intracavitary therapy of malignant pleural effusions [2, 3].
Some required repeated instillations and have been associated with high infection rates $[4,5]$. Our initial experience using a chest tube for intrapleural administration of $\gamma$-IFN in patients with malignant mesothelioma confirmed this finding. Despite daily cleansing of the stoma and rinsing of the tube, infection developed in the majority of cases. To reduce or avoid these problems, we decided to try the totally implantable PAC system.

Use of PACs for access to the peritoneum and the pleural cavity has been described previously [6, 7]. In their report, CRAIG et al. [6] found that the device was a substantial improvement over the Tenckhoff catheter, but deplored a loss of bidirectional flow due to formation of a fibrinous sheath at the tip of the catheter in 11 out of 58 patients. We did not encounter this problem, but it should be stressed that the median duration of placement was 22 weeks in the series of CRAIG et al. [6] as compared to 8 weeks in our study. In any case, a possible solution to this problem would be to inject a small amount of Streptokinase ${ }^{\circledR}$ or Urokinase ${ }^{\circledR}$ into the device in order to dissolve the plugs.

Immediate and complete pleural dispersion usually followed the instillation of the medication. Intracavitary injection of $99 \mathrm{~m}$-technetium-labelled macroaggregates through the PAC showed that instillation was followed by complete and instant diffusion (fig. 3a). Indeed, intrapleural treatment with $\gamma$-IFN did not obliterate the pleural space, and thus pleural distribution remained satisfactory in most patients until the end of treatment (fig. 3b).

By contrast, in another trial using PACs for intrapleural administration of interleukin-2 (IL-2) for patients with malignant mesothelioma, obliteration of the pleural space was common (IL-2 is highly symphysizing), rendering pleural distribution of the drug uneven at the end of treatment. This did not prevent responses, however, which were equally effective with either an open chest tube or the PAC system.

In one patient treated with IL-2, necrosis of the overlying skin necessitated removal of the device. This complication was attributed to local electrontherapy (21 Gy over 3 days), which we routinely carry out after thoracoscopy in patients with malignant mesothelioma, to prevent seeding of tumour along the pathway of the trocar. This complication could have been prevented by implanting the PAC outside the irradiated zone and tunnelling the catheter to the placement site. Tunnelling also minimizes the risk of reflux of the injected product into the pocket. 
The infectious complications that we observed in our first seven patients were probably due to the formation of haematoma around the PAC. This problem was solved by placing a small suction drain in the pocket for a few days.

Using the PAC, local immunotherapy can be an ambulatory procedure. Placement is only a few minutes longer for a PAC than for a chest tube. Patient tolerance is excellent. In our series, most patients did not even feel the device between two treatment sessions. Unlike open chest tubes, which require daily cleansing of the stoma and rinsing of the tube, PACs require minimal maintenance.

Based on our experience, we recommend the PAC system for prolonged administration of intrapleural medication.

\section{References}

1. Boutin C, Viallat JR, Van Zandwijk N, et al. Activity of intrapleural recombinant gamma-interferon in malignant mesothelioma. Cancer 1991; 67: 20332037.

2. Rusch VW, Figlin R, Godwin D, Piantadosi S. Intrapleural cisplatin and cytarabine in the management of malignant pleural effusions: a lung cancer study group trial. J Clin Oncol 1991; 9: 313-319.

3. Montaldo PG, Figoli F, Zanette ML, et al. Pharmacokinetics of intrapleural versus intravenous etoposide (VP 16) and teniposide (VM 26) in patients with malignant pleural effusion. J Clin Oncol 1991; 47: 55-61.

4. Holoye PY, Jeffres G, Dhingra HM, et al. Intrapleural etoposite for malignant effusion. Cancer Chemother Pharmacol 1990; 26: 147-150.

5. Ike O, Shimizu Y, Hitami S, Wado R, Ikada Y. Treatment of malignant pleural effusions with doxorubicin hydrochloride-containing poly (L-Lactic acid) microspheres. Chest 1991; 99: 911-915.

6. Craig EP, Howell SB, Markman M, Lucas WE. Totally implantable system for peritoneal access. J Clin Oncol 1984; 2: 1277-1280.

7. Markman M, Cleary S, Pfeifle C, Howell SB. Cisplatin administered by the intracavitary route as treatment for malignant mesothelioma. Cancer 1986; 58: 18-21. 